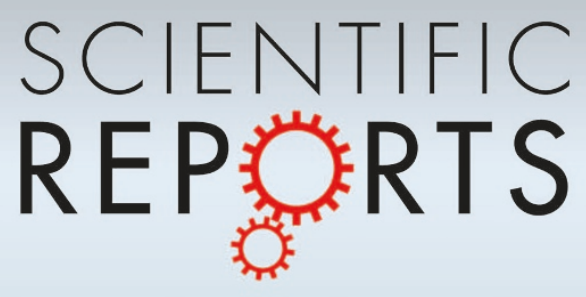

\title{
OPEN Microfluidic control of axonal guidance
}

SUBJECT AREAS:

BIOLOGICAL PHYSICS

NANOSCALE BIOPHYSICS

LAB-ON-A-CHIP

BIOMEDICAL ENGINEERING

Received

24 June 2013

Accepted

14 August 2014

Published

6 October 2014

Correspondence and requests for materials should be addressed to S.M. (smohanty@uta. edu)

* These authors contributed equally to this work.

$\uparrow$ Current address: Dept. of Physics, New York University, NY10012.

\author{
Ling Gu ${ }^{1 *}$, Bryan Black ${ }^{1 *}$, Simon Ordonez ${ }^{1}$, Argha Mondal ${ }^{1} \uparrow$, Ankur Jain² \& Samarendra Mohanty ${ }^{1}$
}

'Biophysics and Physiology Lab, Department of Physics, University of Texas at Arlington, TX76019, ${ }^{2}$ Department of Mechanical and Aerospace Engineering, University of Texas at Arlington, TX 76019.

The precision of axonal pathfinding and the accurate formation of functional neural circuitry are crucial for an organism during development as well as during adult central and peripheral nerve regeneration. While chemical cues are believed to be primarily responsible for axonal pathfinding, we hypothesize that forces due to localized fluid flow may directly affect neuronal guidance during early organ development. Here, we report direct evidence of fluid flow influencing axonal migration, producing turning angles of up to $90^{\circ}$. Microfluidic flow simulations indicate that an axon may experience significant bending force due to cross-flow, which may contribute to the observed axonal turning. This method of flow-based guidance was successfully used to fasciculate one advancing axon onto another, showcasing the potential of this technique to be used for the formation of in vitro neuronal circuits.

T he process of synaptogenesis ${ }^{1}$ requires newly developed axonal terminals to find precise synaptic partners over complex pathways and tremendous distances (up to one meter). While chemical gradients have been widely studied and heavily implicated in all stages of neuro- and synaptogenesis, their description remains incomplete, and there has been a recent shift in the discussion to other potential contributors to the neural developmental landscape. It is well known that fluid flow is involved in various processes during organogenesis ${ }^{2}$. Chemical and shear stress signalling information is carried by fluid flows, and fluid-dependent mechanical stress mediates kidney morphogenesis ${ }^{3}$, cardiovascular development ${ }^{4}$, and even neuron cell migration ${ }^{5}$. It has been shown that cerebrospinal fluid flow generated by beating of the ependymal cilia is a requirement for the directional migration of new neuroblasts toward the olfactory bulb ${ }^{6}$. However, to date, no evidence exists to confirm the direct influence of microfluidic flow on axonal outgrowth and migration. Furthermore, it is of significant interest to evaluate effect of microfluidic flow since lab-on-a-chip devices ${ }^{7}$ are finding an increased role in axonal migration and regeneration studies ${ }^{8}$; a burgeoning field of research due to its importance in the study of functional neuronal circuitry.

Our recent studies have indicated that axons can be effectively guided by laser-driven vaterite micromotors, which we believe create a microfluidic flow-induced localized shearing effect in the region near the growth cone? Although shear stress was believed to be the primary driving mechanism for axonal guidance, the possibility of direct mechanical forcing of filopodia by the micromotor could not be completely ruled out, and the release of calcium-ions from the rotating birefringent particle (if any by solubilisation) might have also directly affected the growth cone. Herein, we study the influence of direct laminar microfluidic flow and report the highly effective guidance of primary goldfish retinal ganglion cell axons. Utilizing this method, growth cones could be guided over long ranges (both angle and distance), thus illustrating a persistent and unambiguous role of fluid flow during neurogenesis and allowing the formation of an in vitro neuronal circuit.

\section{Results}

For studying the effects of microfluidic flow on axonal migration, a micro-syringe nano-pump was used to direct cell culture medium towards the axons of retinal ganglion cells (RGC), derived from common goldfish (Carassius auratus). As shown in Fig. 1a (and Suppl. Fig. 1a), the microfluidic flow created by the micro-syringe pump was introduced into the sample chamber (SC) via micro-tubing (inner diameter: $50 \mu \mathrm{m}$ ). The outflow tip of the micro-tubing was placed $\sim 100 \mu \mathrm{m}$ away from the growth cone, approximately perpendicular to the outgrowth direction. The flow rate was fixed at $2.5 \mu \mathrm{L} / \mathrm{min}$ for the entire experimental duration (between 40 and $80 \mathrm{~min}$ ). Of the actively growing axons $(n=23), 35 \%$ of growth cones were found to respond to microfluidic flow (Suppl. Fig. $1 \mathrm{~b}$ ), very similar to the $\sim 40 \%$ turning events observed in response to the rotating micromotor approach ${ }^{9}$. Out of these flow-responsive growth cones $(\mathrm{n}=8), 78 \%$ followed the fluid-flow direction, while $22 \%$ grew against the flow. Fig. 1b shows a histogram of relative turning angles in the absence (negative control) and presence of microfluidic flow at two different time points $(\mathrm{n}=8)$. As compared to control growth experiments $\left(5.5 \pm 1.5^{\circ}\right)$, where no flow was applied, statistically significant $(\mathrm{p}=0.006)$ fluid flow effects were observed. The average 


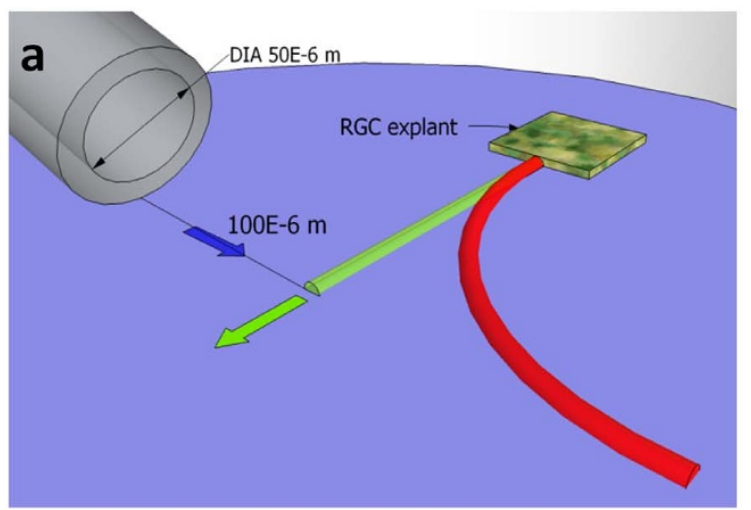

b
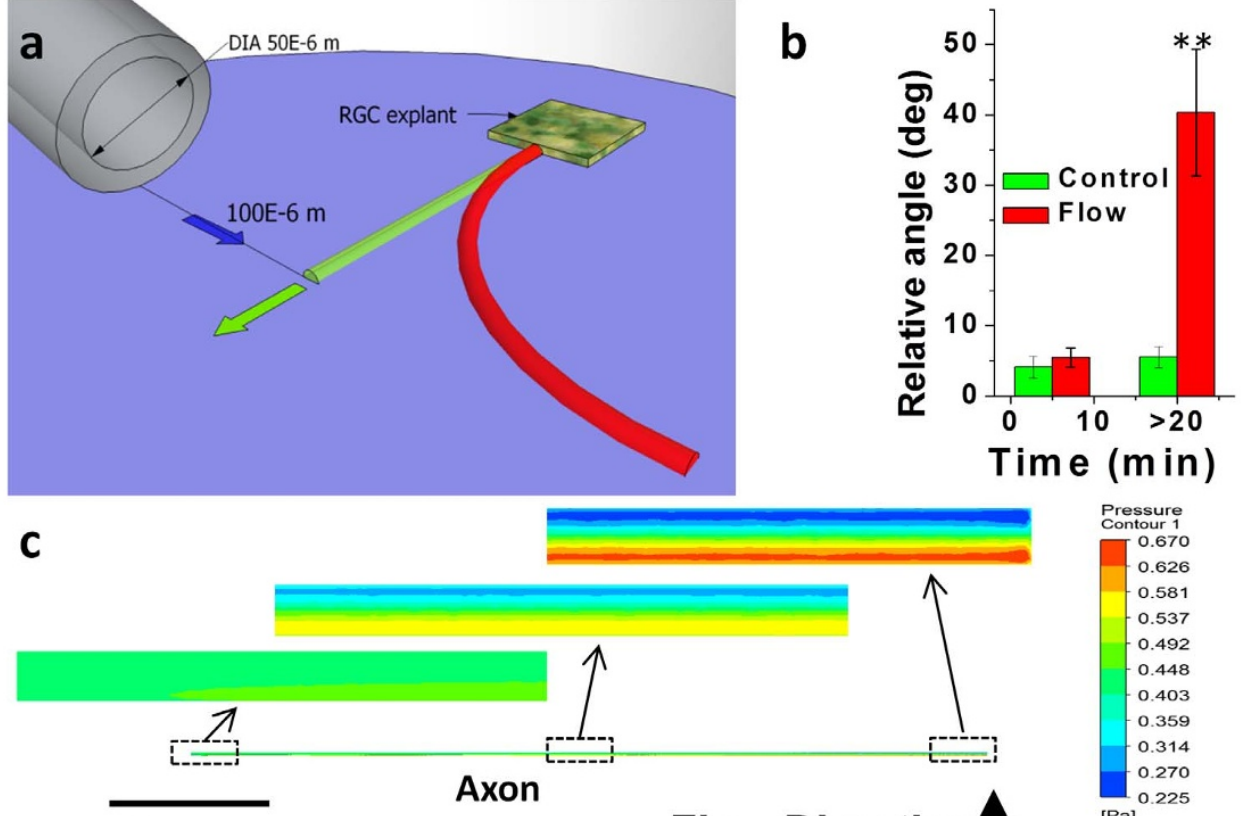

$20 \mu \mathrm{m}$

Flow Direction $\uparrow$

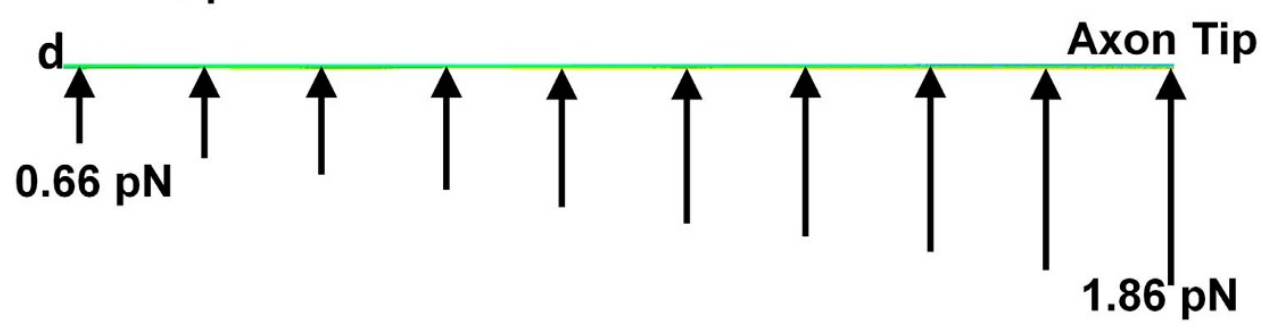

Figure $1 \mid$ (a) Petridish-view of microfluidic guidance setup. Green cylinder indicates initial orientation of axonal and growth cone, while green arrow indicates initial outgrowth direction. Red cylinder indicates final orientation of axonal shaft and growth subsequent to application of microfluidic flow (Blue arrow: flow rate: $2.5 \mu \mathrm{L} / \mathrm{min}$ ). (b) Histogram of axonal turning angle in absence (control) and presence of microfluidic flow at two different time points $(n=8)$. The error bars around mean represent standard error of the mean. (c) Simulation of radial force distribution on axon induced by microfluidic flow at various axial positions, (d) estimated total force on axonal elements induced by microfluidic flow along length of axon. Magnitude of force is depicted by length of arrow.

relative turning angle due to the application of fluid flow was found to be $40.4 \pm 9.0^{\circ}$. This $n$-number represents axons derived from multiple fish $(\mathrm{n}=7)$. While the axon advances stochastically, elongating by binding single finite unit molecules to the former chain of tubulin, its response to fluid flow is immediate in the sense that each unit will respond immediately to the force acting on it. However, the axon is a highly polarized and surprisingly rigid structure, so the fluid-flow response is both immediate and permanent (in the absence of other forces). It may be noted that when the axon grows towards the axial centre of the fluid flow, the loading force increases and the axonal response is not an immediate response, in the sense that its initial and final state differ in time.

While filopodia on the growth cone are known to be critical elements of axonal guidance, simulating the fluid flow effects on individual filopodium and physico-chemical signal transduction and integration over the surface of a growth cone is very complex. Therefore, for the purposes of this first-order simulation of the effects of fluid flow on axonal migration, we have restricted ourselves to the external fluid flow forces on the axon itself. To determine the total force exerted by the flow on the axon, finite-element simulation of the microfluidic flow was carried out in ANSYS-CFX. The axon was modelled as a semi-cylinder of $0.5 \mu \mathrm{m}$ diameter lying flat on the bottom surface of the sample chamber, with cross-flow originating from an opening at a distance of $100 \mu \mathrm{m}$ from the axon axis. The total force induced on the axon was determined by integrating the net pressure and shear stress profiles on the axon surface over a length of $100 \mu \mathrm{m}$. The total force acting on a $100 \mu \mathrm{m}$ long, $0.5 \mu \mathrm{m}$ diameter axon in $2.5 \mu \mathrm{L} / \mathrm{min}$ cross-flow was found to be $12.5 \mathrm{pN}$. Fig. 1c shows a top view of the pressure profile on the axon surface, indicating higher pressure upstream of the axon compared to downstream, leading to a net bending force in the direction of flow. Fig. 1d shows the flow-induced bending force distribution on the axon along its length, obtained by axially dividing the axon into a number of sections and axially integrating the pressure and shear stress distributions in each section to determine the net force in each section. Suppl. Fig. 2a shows a plot of the transverse bending force as a function of flow rate, indicating that the bending force increases linearly with flow rate. Simulations also showed that the bending force is a function of distance between the axon and the flow opening. The total force increases from $12.6 \mathrm{pN}$ to $20.7 \mathrm{pN}$ as the distance is reduced from $100 \mu \mathrm{m}$ to $50 \mu \mathrm{m}$. Suppl. Fig. $2 \mathrm{~b}$ shows the variation in bending force as a function of axon diameter. For fixed flow rate, the bending force increases significantly as the axon diameter increases.

In order to examine if microfluidic flow can deviate an axon from establishing fasciculation with another axon, two axons migrating in orthogonal direction were selected. Then, the micro-tubing was oriented at an angle of approximately $120^{\circ}$ with respect to the axonal outgrowth direction. Figs. 2.a to f show the time-lapse images of significant deviation of the growth cone direction of one axon (with respect to the other axon) achieved by microfluidic flow. The sequence of pseudocolor overlay profiles (Figs. $2 \mathrm{~g}$ to i) clearly depict 


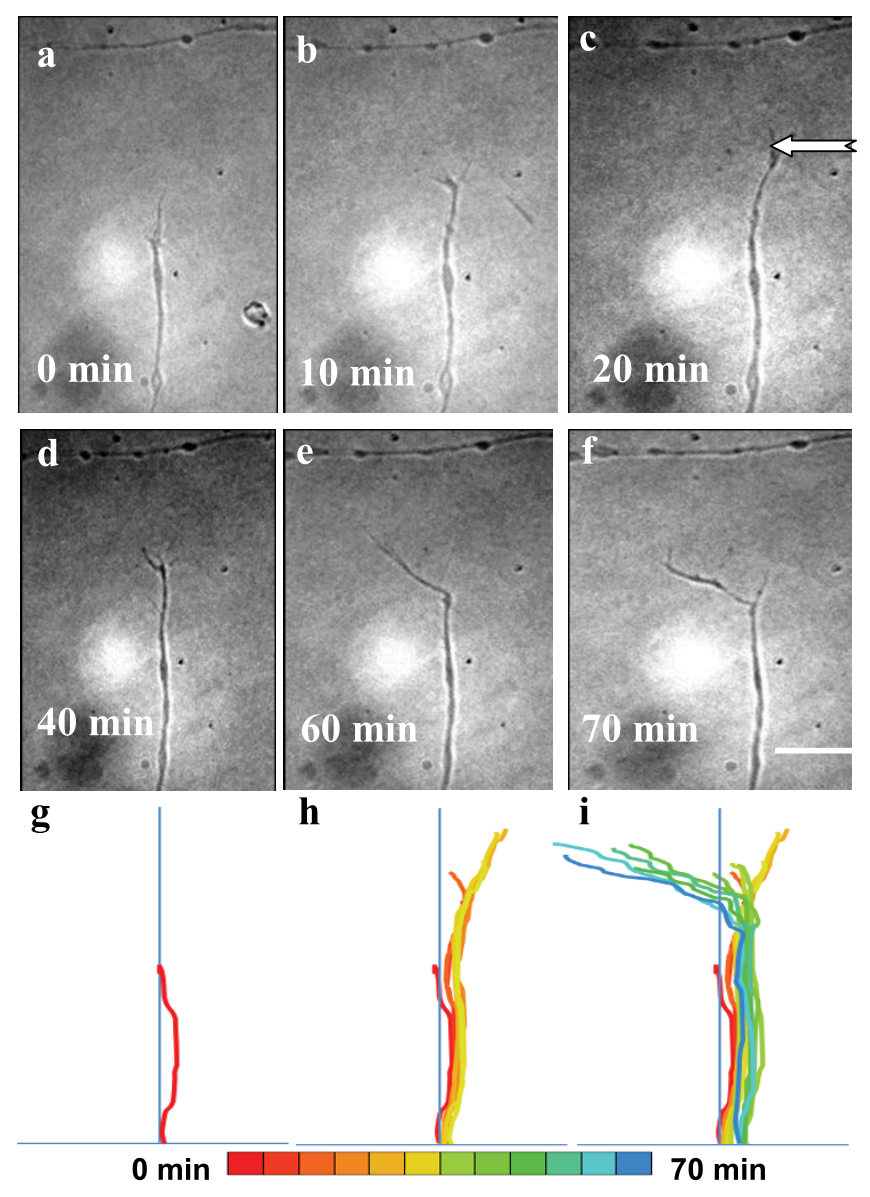

Figure $2 \mid(a-f)$ Time-lapse images showing significant deviation of the direction of growth cone migration in response to microfluidic flow. The direction of flow is marked by white arrow. Bar: $20 \mu \mathrm{m}$. (g-i) Sequence of overlay profiles depicting the directional change of axonal growth in pseudocolor from 0 to $70 \mathrm{~min}$.

this directional change. In some cases, the application of fluid flow at large angles $\left(\sim 140^{\circ}\right)$ resulted in neuronal growth cone migration in the opposite to the microfluidic flow. Suppl. Fig. 3 (a to i) shows the time-lapse images of growth cone turning against the direction of microfluidic flow after continuous $45 \mathrm{~min}$ of flow application. Since the direction of flow has a larger angle $\left(\sim 140^{\circ}\right)$ with the original growth direction, it seems difficult for the axon to follow the flow direction (as compared to $120^{\circ}$ in Fig. 2). Instead of turning to the direction of flow, it preferred to grow against the direction, which may help in minimizing the cross-sectional shear force on the axon generated by the flow. However, this purely physical interpretation is obviously incomplete as it does not account for the chemical or biological complexities of the fluid-flow interaction. Further studies will be required to more rigorously address the underlying mechanism for this unanticipated result. But it is important to reiterate that $78 \%$ of the growth cones which were found to respond followed the direction of the fluid flow. Growth cones with higher growth rates were found to be more responsive to the microfluidic flow.

In some cases, where microfluidic flow was applied at larger angles with respect to the axonal shaft, directional reversal of axonal migration was observed, though accompanied by slow after-turn growth. In Suppl. Fig. 4, we show the time-lapse images of growth cone response to application of microfluidic flow at $\sim 140^{\circ}$. In this case, an axon turning downward (Suppl. Fig. 4a), retracts as evident by formation of retraction bulb (Suppl. Fig. 4b, blue arrow shows the direction of retraction) after $10 \mathrm{~min}$ of microfluidic flow application. Retraction of axon continued for $30 \mathrm{~min}$ (Suppl. Figs. $4 \mathrm{~b}$ to d), dur- ing which the retraction bulb was found to fuse with an axonal bulb (Suppl. Fig. 4d). Emergence of growth cone is shown in Suppl. Fig. 4e. Suppl. Figs. 4e to $\mathrm{f}$ show turning and growth of the axon nearly parallel to the flow direction (blue arrow shows the direction of growth). In such case, the growth rate after the turning event was found to decrease indicating loss of growth capability of the axon due to detachment by significant fluid force application.

Next, we determined the kinetics of axonal growth rate and turning angle in response to microfluidic flow $(n=8)$. Average kinetics of advancing axon's turning angle is shown in Fig. 3 (a). While the turning angle progressively increased with increasing durations of microfluidic flow application, at 40 min the average turning angle decreased, but again increased back to the average saturated turning angle of $\sim 30^{\circ}$. The average turning rate was $0.825 \pm 0.781$ degrees per minute, while the turning axons continued to advance at an average growth rate of $0.301 \pm 0.244 \mu \mathrm{m} / \mathrm{min}$ (Fig. $3 \mathrm{~b}$ ). Though the growth rate decreased after $25 \mathrm{~min}$ (Fig. 3b), it was not statistically significant. In Fig. 3c, we show the cumulative distribution of turning (angle) of the axon during microfluidic flow. The variability observed in the turning kinetics (Fig. 3a) and growth rate (Fig. 3b) is owing to the fact that not only the axons from different neuronal cells can be in different physiological state; there is a distribution of axonal diameter. As shown in Suppl. Fig. 2b, the total estimated force at a fixed flow rate is higher for axons with larger diameters. Albeit the axons having larger diameters consist of larger number of microtubules which is expected to lead to lower bending angle for fixed applied force. Therefore, we also carried out numerical simulation on the effect of force of fluid flow on axonal bending. Force simulations were based upon drag force calculations where the axon was idealized to be a semicircular cylinder, with its leading edge positioned along the microfluidic tube's axis, at a separation of $100 \mu \mathrm{m}$. The axon was then treated as a bundle of different number of microtubules ${ }^{10}$, linearly adding their flexural rigidity. The force distribution on the axon along its length (Fig. 1d) was taken as input to estimate the flow-induced bending. Simulations of this calculated drag force led to 'bending' of the axonal shaft with different final angles for 20, 40, 60, 80 and 100 microtubules. Fig. $3 d$ shows the 'bent' axon due to microfluidic flow versus the initial direction of the axon's growth. Suppl. Fig. 5a shows simulated bending radius of axons as a function of different number of microtubules for the force field shown in Fig. 1d. Suppl. Fig. 5b shows simulated final turning angle as a function of number of microtubules for fixed fluid-force.

Finally, we went on to turn one of the two parallel growing axons to fasciculate on the other axon by microfluidic flow. Fig. 4a shows an axon whose growth direction is initially perpendicular to the fluid flow direction (indicated by red arrow). Within ten minutes, the growth cone direction started turning to the right $\left(20^{\circ}\right.$, Fig. $\left.4 \mathrm{~b}\right)$, towards the fluid flow direction. The growth cone continues turning in the fluid flow direction from $0^{\circ}$ to $90^{\circ}$ (Fig. 4 a-e, Suppl. Movie 1) until it merges (fasciculates) with another axon (Fig. 4e-h). The overlay pseudocolor profiles in Fig. 4i show the axonal turning and fasciculation process. Interestingly, the growth cone of the guidedaxon was observed to split into two opposite directions along the second axon shaft. The sequence of overlays, in intervals of every $5 \mathrm{~min}$, is shown in Suppl. Fig. 5c. The turning angle is found to increase linearly with time (Fig. $4 \mathrm{j}$ ).

Since the axon was made to take sharp turn (Fig. 4 a to $\mathrm{j}$ ) by the microfluidic flow, health of guided axon was evaluated by monitoring the growth rate. Prior to the application of fluid flow, the axonal growth rate was $1.4 \pm 0.63 \mu \mathrm{m} / \mathrm{min}$, which is consistent with previous measured values ${ }^{11}$. The growth rate during guidance experiments decreased to $0.301 \pm 0.244 \mu \mathrm{m} / \mathrm{min}$. The differences in the growth values shown in Fig. $4 \mathrm{k}$ are statistically significant between initial (10 $\mathrm{min})$ turning and later time point (20 $\mathrm{min})$. However, it is not statistically significant between later time points (35 $\mathrm{min}$ ). 
a

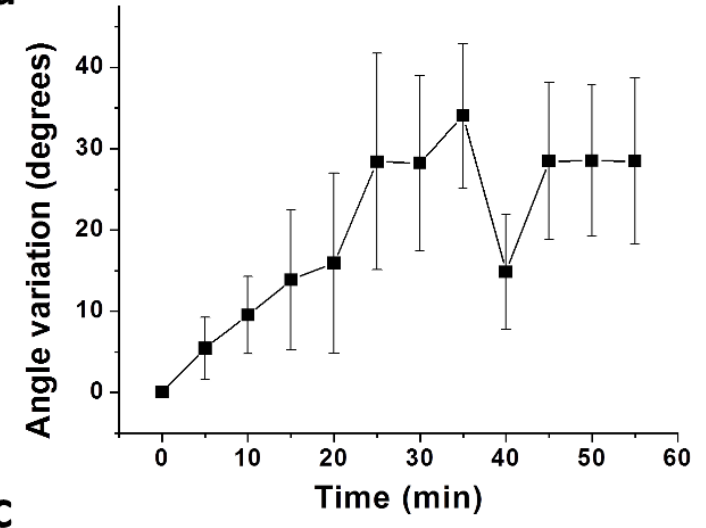

C

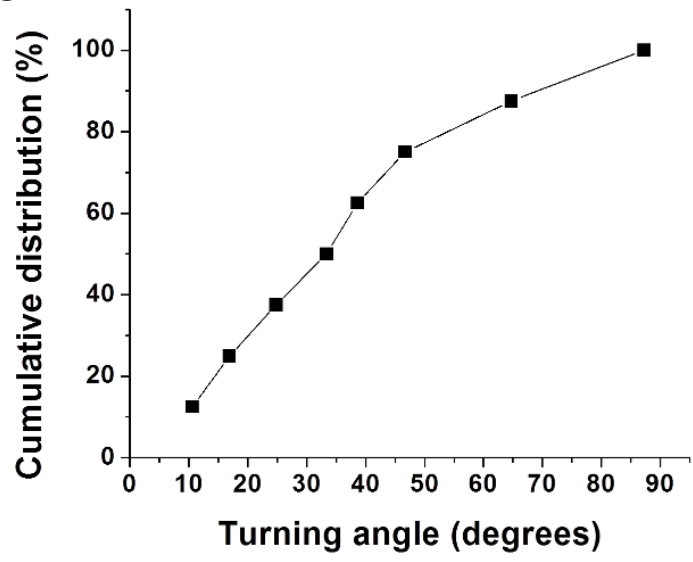

b
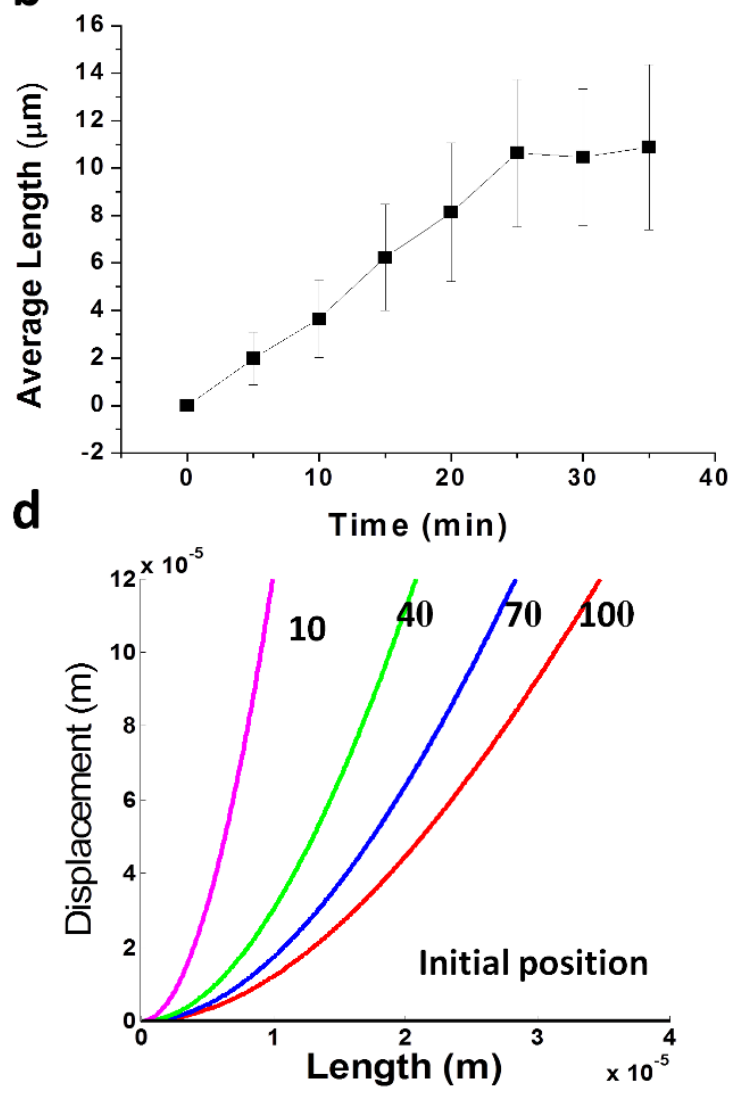

Figure 3 (a) Average kinetics of advancing axon's turning angle in response to microfluidic flow $(\mathrm{n}=8)$. (b) Axonal growth kinetics during microfluidic guidance $(n=8)$. The error bars around mean represent standard error of the mean. (c) Cumulative distribution of turning (angle) of the axon during microfluidic flow $(\mathrm{n}=8)$. ( $\mathrm{d}$ ) Theoretically predicted final position of axon after bending under application of a distributed force for an axon having 10, 40, 70 and 100 microtubules.

However, the growth rate did not change significantly after the turning, as shown in Fig. 4k. Further, while axon growth is always stochastic, presence of other axon can be sensed by filopodia of the turning axon and thus, can modulate its growth rate. The fact that axons still advance after such large turning angles are induced by the fluid flow shows that the guidance event is not, in itself, damaging to the axon, and allows the axon to grow at normal rates. However, it is necessary to point out that among the population of axons which did not respond to the fluid flow, several $(n=6)$ retracted (example in Suppl. Fig. $6 a-d)$, or detached $(n=2)$ from the dish entirely (Suppl. Fig. 6e-h). This leads us to believe that a future, more parameterized study may reveal an even more efficient flow rate and micro-tubing separation threshold. In this case, the neuronal guidance realized by our microfluidic approach can have potential application in fabrication of in vitro neuronal circuits on a siliconbased stimulation and/or detection devices. Later, with continuous application of fluid flow, while the final turning axonal angle was still $90^{\circ}$, the axonal shaft curved around the corner (Fig. 4h and Suppl. Movie 1). This may be attributed to axonal microtubules having to undergo much larger stress levels in order to bend with a small radius of curvature.

\section{Discussion}

Bending of the axonal shaft often show an abrupt "kink" in the shaft, suggesting that the contour is caused by something other than simple bending. It is well established that when sufficiently large forces act on a microtubule or collection of microtubules, they are subject to breaking $^{12}$. This is in part due to the elastic energy stored in the microtubule lattice, which has been found to be surprisingly rigid.
The observed sharp and high turning angles observed during microfluidic flow imply that microtubules may break and are reorganized into non-centralized microtubules. It must be noted that though we clearly demonstrated the effect of fluid flow in migration of primary axons, the complexity of the underlying biological processes can, in no way, be undermined. The growth cone processes responsible for directionality of growth are rather complex and considering the influence of external microfluidic forces on these processes would require further biochemical studies and biophysical modelling of the mechanotransduction process. Moreover, in in-vivo conditions, cell and axon migration is typically through a three-dimensional matrix, and it has been shown that certain growth and migration properties vary between two- and three-dimensional environments ${ }^{13}$.

There are several known organ-specific fluid flow-related diseases (e.g. ciliopathies and atherosclerosis), and the loss of fluid flow during neurogenesis has been shown to lead to fluid accumulation, which can lead to distention pathologies in the brain ${ }^{6}$. Our results suggest that forces due to microfluidic flow are sufficient to significantly modify the direction of axonal migration over large turning angles and long distances. This can is important for developmental, as well as adult axonal regeneration-migration studies. One possible mechanism behind this flow-induced turning event is that shear stress increases the intracellular calcium level by stimulation of stretch-activated (mechanosensitive) calcium ion channels, since changes in intracellular calcium concentration is known to regulate growth cone guidance ${ }^{14}$. In addition to direct activation, it has been shown that mechanosensitive TRPV4 ion channels can stimulate binding of additional $\beta 1$ integrin receptors, and promote remodelling of the cytoskeleton and ultimately, reorientation of the cell ${ }^{15}$, and that integrin signalling is implicated in the directional migration 

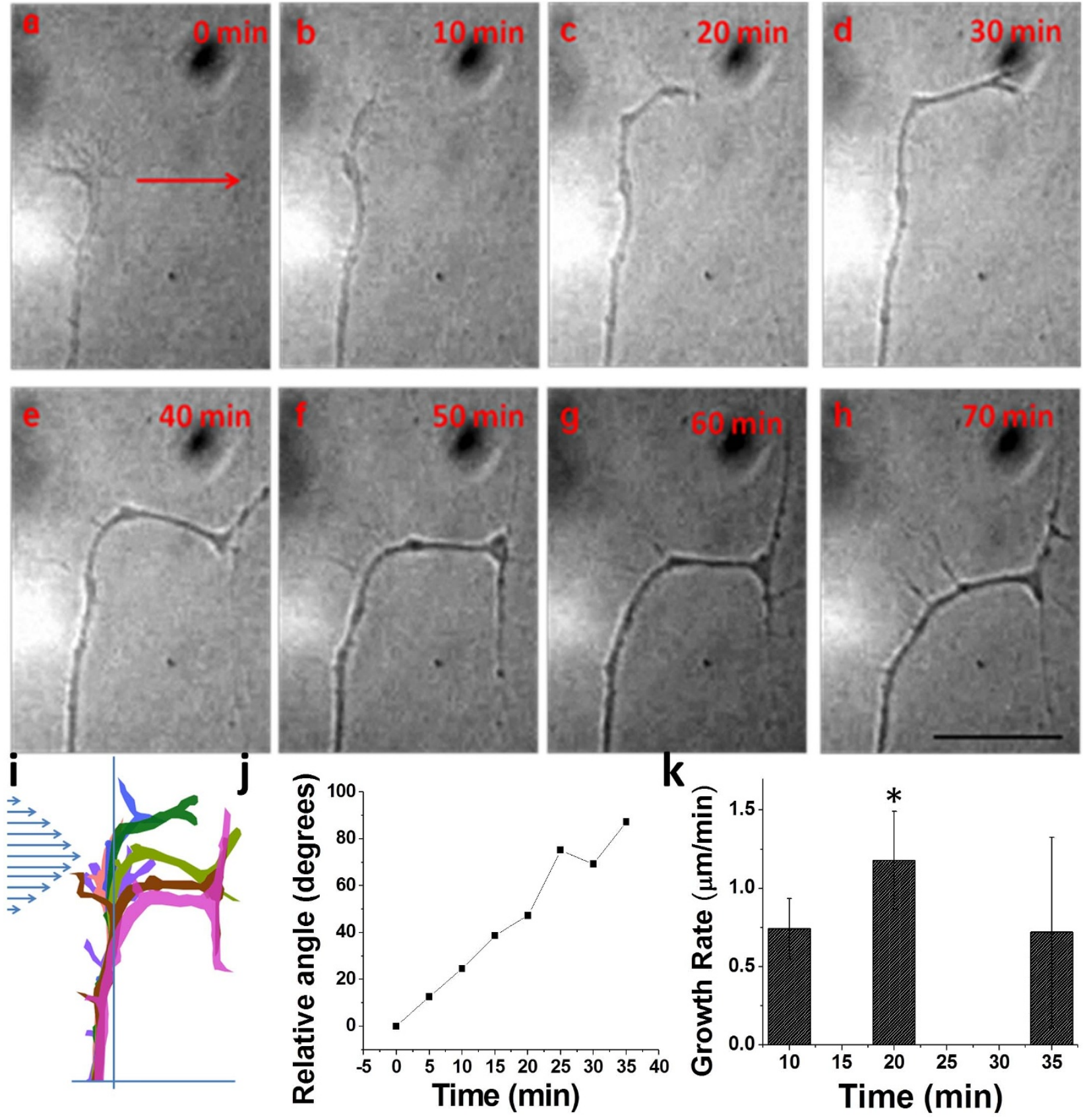

Figure $4 \mid$ (a-e) Time-lapse images of turning of growth cone in response to direct microfluidic flow. The direction of flow is marked by red arrow, the angle between original growth direction and flow direction being $\sim 90^{\circ}$. (f-h) Fasciculation of guided axon over another axon. Scale bar: $50 \mu \mathrm{m}$.

(i) Overlapped outline of microfluidic flow assisted axonal turning and fasciculation process. Vertical axis represents initial outgrowth direction. Blue arrows illustrate fluid flow profile. (j) Kinetics of turning (angle) of the growth cone during microfluidic flow. (k) Growth rate of axon at different time points during turning.

of breast carcinoma cells due to creeping fluid flow ${ }^{16}$. Further, autocrine signaling ${ }^{17}$, modulated by flow, has been implicated to influence tumor cell migration and the observed microfluidic axonal guidance may be mediated by such signalling mechanism. Despite these advancements in our fundamental understanding of contributions due to fluid flow, demonstrating such effects in-vivo may be extremely difficult due to the complexity of in-vivo fluid flow and the contribution from chemical information contained in the fluid flow which acts in microscopic spatial and temporal regimes ${ }^{18}$.

\section{Conclusions}

To conclude, neuronal growth cones were observed to respond to direct microfluidic flow generated by a microfluidic pump. The lin- ear microfluidic flow, introduced by microsyringe pump, was found to be an effective approach in directing the axonal growth. The microfluidic flow could enable formation of neuronal fasciculation and also for hindering it. It remains to be evaluated if microfluidicmechanosensing by retinal ganglion cell axons can be extended to other types of neurons, which can provide new insights into sensing mechanism by neuronal growth cones. Microfluidic lab-on-a-chip devices can increase the throughput of the axonal guidance process so as to allow formation of in-vitro neural circuits. The microfluidic flow-controlled axonal guidance method has potential to be advantageously employed to study regeneration subsequent to nerve damage, which is relevant to the situation encountered in vivo in spinal cord injury ${ }^{19}$. 


\section{Methods}

Ethics statement. All experimental procedures were conducted according to the University of Texas at Arlington-Institutional Animal Care and Use Committee approved protocol A10.010.

Explant culture. Retinal ganglion cells of common goldfish, Carassius auratus, were used in these studies ${ }^{20}$. Adult fish, 5-7 $\mathrm{cm}$ body length, were housed in standard glass aquaria at $19^{\circ}-21^{\circ} \mathrm{C}$. In order to facilitate axonal outgrowth in culture, fish received a priming lesion of the optic nerve at least 1 week before removing the retina ${ }^{20}$.

Following anesthesia with tricaine methanesulfonate (Argent), the optic nerve was visualized through an incision in dorsal-posterior conjunctiva and crushed with Dumont \#5 forceps distally where the nerve exits the orbit. Care was taken to avoid damaging the large blood vessel which runs alongside the nerve. One to 2 weeks postcrush, eyes were removed from the anesthetized goldfish after one hour of dark adaptation to facilitate removal of the pigment epithelium. The retinas were removed and cut into $300 \mu \mathrm{m}$ square explants on a Mcllwain tissue chopper. The explants were then placed onto glass coverslip previously coated with poly-D-lysine (Sigma, $>300,000 \mathrm{MW}$ in borate buffer, $\mathrm{pH}$ 8.3). The explants were oriented ganglion cell side towards the coverslip, and incubated at room air and temperature in Leibovitz's L15 medium (Gibco) supplemented with 10\% fetal bovine serum (Fisher Scientific) and $50 \mu \mathrm{g} / \mathrm{ml}$ gentamicin. Since goldfish are poikilothermic, it is easy to maintain the retina in the Petri dishes at room temperature and visualize axonal growth under the microscope.

Set up for axonal navigation. The schematic of the platform for microfluidic axonal guidance is shown in Fig. 1. The retina explant in the culture platform was placed on an inverted microscope (Ti-U Eclipse, Nikon). To achieve microfluidic guidance, a micro-tubing (inner diameter: $50 \mu \mathrm{m}$ ) was introduced into the sample chamber (SC) and flow was created by a micro-syringe pump. The outflow tip of the micro-tubing was placed $\sim 100 \mu \mathrm{m}$ away from the growth cone, positioned almost orthogonally to the axonal shaft, unless mentioned specifically.

Simulation of microfluidic flow induced force on axon. Finite-element simulation of the microfluidic flow was carried out in ANSYS-CFX to determine the flowinduced force on the axon. While uniform flow over an infinitely long semi-cylinder has been modelled analytically in the past, in this case, the axon is finite in length, and the incoming flow originates from a circular opening. As a result, an analytical flow solution for this problem is difficult, and one must rely on a simulation model to determine bending axon on the axon. The axon was modelled as a semi-cylinder of fixed diameter lying flat on the bottom surface of the sample chamber, with cross-flow originating from an opening at a fixed distance from the axonal shaft. The fluid inlet was positioned orthogonally to the axonal shaft. Due to the small axon size relative to other geometries in the model, more than 2.7 million nodes were used for meshing. Force induced on the axon was determined by integrating the pressure and shear stress distributions on the axon surface predicted by the simulation model.

Theoretical calculation of fluid-flow force induced axonal bending. Microtubules are long filaments, which serve as tracks for intercellular motor proteins and facilitate the structural functions in eukaryotic cells. In a typical axon, $\sim 50$ such microtubules are assumed ${ }^{10}$ to be present in any given axonal shaft cross-section. The axonal growth cone polymerization processes are modulated asymmetrically by the shearforce generated by the microfluidic flow. To analyze the deformation, or bending, of an axon due to force over its distal end $(25 \mu \mathrm{m})$, we modelled the axon as a beam of regular and uniform cross-section, made of a linear elastic material (microtubule with known ${ }^{10}$ flexural rigidity) that is homogeneous and isotropous with the load concentrated at the axonal terminal. The methodologies used to quantify these deflections can basically be categorized into analytical or finite element analysis techniques. The analytical method is based on Euler-Bernoulli's (EB's) theory, which provides a means to quantify such deflections. Then, the finite element method is utilized to obtain the solution of the bending radius of curvature of an axonal shaft under constant force at the tip. Details described in supplementary methods.

1. Kwon, H. B. et al. Neuroligin-1-dependent competition regulates cortical synaptogenesis and synapse number. Nat Neurosci 15, 1667-+ (2012).

2. Cartwright, J. H. E., Piro, O. \& Tuval, I. Fluid dynamics in developmental biology: Moving fluids that shape ontogeny. HFSP Journal 3, 77-93 (2009).

3. Serluca, F. C., Drummond, I. A. \& Fishman, M. C. Endothelial signaling in kidney morphogenesis: A role for hemodynamic forces. Curr Biol 12, 492-497 (2002).

4. Slough, J., Cooney, L. \& Brueckner, M. Monocilia in the embryonic mouse heart suggest a direct role for cilia in cardiac morphogenesis. Dev Dynam 237, 2304-2314 (2008).
5. Sawamoto, K. et al. New neurons follow the flow of cerebrospinal fluid in the adult brain. Science 311, 629-632 (2006).

6. Kramer-Zucker, A. G. et al. Cilia-driven fluid flow in the zebrafish pronephros, brain and Kupffer's vesicle is required for normal organogenesis. Development 132, 1907-1921 (2005)

7. Taylor, A. M. et al. A microfluidic culture platform for CNS axonal injury, regeneration and transport. Nat Methods 2, 599-605 (2005).

8. Kim, Y.-T., Karthikeyan, K., Chirvi, S. \& Dave, D. P. Neuro-optical microfluidic platform to study injury and regeneration of single axons. Lab on a Chip $\mathbf{9}$, 2576-2581 (2009).

9. Wu, T. et al. A photon-driven micromotor can direct nerve fibre growth. Nat Photon 6, 62-67 (2012)

10. Peter, S. J. \& Mofrad, M. R. Computational modeling of axonal microtubule bundles under tension. Biophys J 102, 749-757 (2012).

11. Bastmeyer, M., Beckmann, M., Schwab, M. \& Stuermer, C. Growth of regenerating goldfish axons is inhibited by rat oligodendrocytes and CNS myelin but not but not by goldfish optic nerve tract oligodendrocytelike cells and fish CNS myelin. The Journal of Neuroscience 11, 626-640 (1991).

12. Odde, D. J., Ma, L., Briggs, A. H., DeMarco, A. \& Kirschner, M. W. Microtubule bending and breaking in living fibroblast cells. J Cell Sci 112, 3283-3288 (1999).

13. Meyer, A. S. et al. 2D protrusion but not motility predicts growth factor-induced cancer cell migration in 3D collagen. The Journal of Cell Biology 197, 721-729 (2012).

14. Gomez, T. M. \& Spitzer, N. C. Regulation of growth cone behavior by calcium: new dynamics to earlier perspectives. J Neurobiol 44, 174-183 (2000).

15. Thodeti, C. K. et al. TRPV4 Channels Mediate Cyclic Strain-Induced Endothelial Cell Reorientation Through Integrin-to-Integrin Signaling. Circ Res 104, 1123-U1278 (2009).

16. Polacheck, W. J., Charest, J. L. \& Kamm, R. D. Interstitial flow influences direction of tumor cell migration through competing mechanisms. P Natl Acad Sci USA 108, 11115-11120 (2011).

17. Shields, J. D. et al. Autologous chemotaxis as a mechanism of tumor cell homing to lymphatics via interstitial flow and autocrine CCR7 signaling. Cancer Cell 11, 526-538 (2007).

18. Freund, J. B., Goetz, J. G., Hill, K. L. \& Vermot, J. Fluid flows and forces in development: functions, features and biophysical principles. Development 139, 1229-1245 (2012).

19. Fawcett, J. Repair of spinal cord injuries: where are we, where are we going? Spinal Cord 40, 615-623 (2002).

20. Landreth, G. E. \& Agranoff, B. W. Explant culture of adult goldfish retina: a model for the study of CNS regeneration. Brain Res 161, 39-55 (1979).

\section{Acknowledgments}

S.M. would like to thank the support from Office of President and Provost, The University of Texas at Arlington and the National Science Foundation (1148541).

\section{Author contributions}

L.G. and S.O. performed cortical neuron culture. L.G., S.O., B.B. and S.M. performed the experiments and analyzed the data. A.M. developed the axonal bending model and A.J. developed the microfluidic flow simulation model. S.M. designed and supervised the project. All authors wrote the paper.

\section{Additional information}

Supplementary information accompanies this paper at http://www.nature.com/ scientificreports

Competing financial interests: The authors declare no competing financial interests.

How to cite this article: Gu, L. et al. Microfluidic control of axonal guidance. Sci. Rep. 4, 6457; DOI:10.1038/srep06457 (2014).

This work is licensed under a Creative Commons Attribution-NonCommercialShareAlike 4.0 International License. The images or other third party material in this article are included in the article's Creative Commons license, unless indicated otherwise in the credit line; if the material is not included under the Creative Commons license, users will need to obtain permission from the license holder in order to reproduce the material. To view a copy of this license, visit http:// creativecommons.org/licenses/by-nc-sa/4.0/ 